Supporting Information

\title{
Cobalt Phosphates Loaded into lodine-Spaced Reduced Graphene Oxide Nanolayers for Electrochemical Measurement of Superoxide Generated by Cells
}

ZhuoZou ${ }^{\dagger, \ddagger, \#, ~ J i e ~ C h e n ~}{ }^{\dagger, \#}$, Zhuanzhuan Shis,\#, Chengsong Yuan",\#, Guangdong Zhou ${ }^{\dagger}$, Qian Liu ${ }^{\dagger}$, Hong Chen, Qingxin Zeng ${ }^{\dagger}$, Taotao Liang ${ }^{\dagger}, "$, Kang Lai Tang ${ }^{*}, "$ and Chang Ming $\mathrm{Li}^{*},+, \S, \perp$

† Chongqing Engineering Research Center for Rapid Diagnosis of Dread Diseases School ofMaterials and Energy, Southwest University, Chongqing 400715, China ‡ Faculty of Psychology, Southwest University, Chongqing 400715, China $\S$ Institute of Materials Science and Devices, School of Materials and Engineering, Suzhou University of Science and Technology, Suzhou 215011, China

"Department of Orthopedic Surgery, Southwest Hospital, Army Medical University, Chongqing 400038, China

${ }^{\perp}$ Institute of Advanced Cross-field Science, College of Life Science, Qingdao University, Qingdao 266071, China

*Email: ecmli@swu.edu.cn.

*Email: tangkanglai@hotmail.com. 

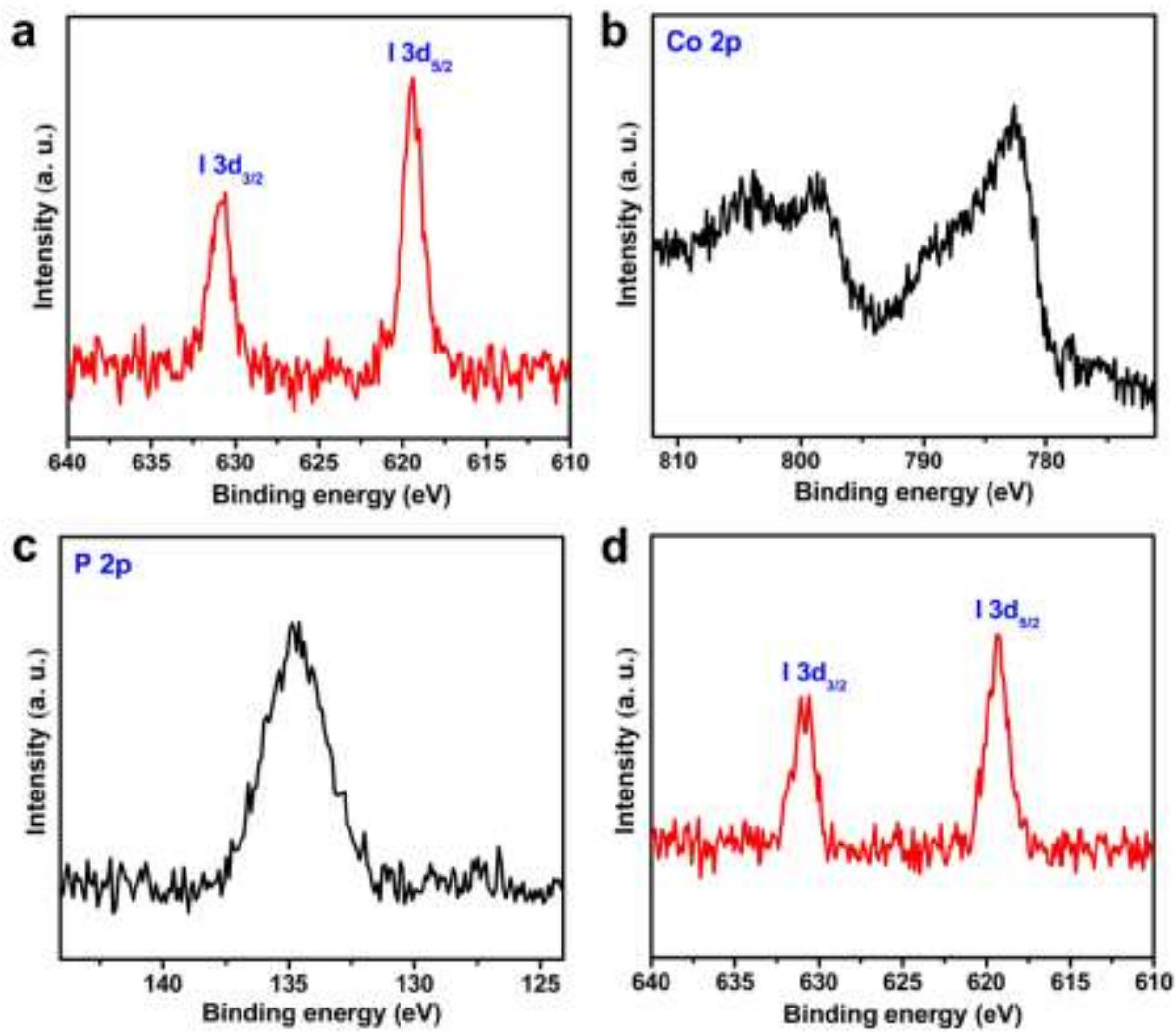

Figure S1. XPS analysis about (a) I 3d spectra in I-rGO, (b) Co 2p spectra in $\mathrm{Co}_{3}\left(\mathrm{PO}_{4}\right)_{2} / \mathrm{rGO}$, (c) P 2p spectra in $\mathrm{Co}_{3}\left(\mathrm{PO}_{4}\right)_{2} / \mathrm{rGO}$, and (d) I 3d spectra in $\mathrm{CO}_{3}\left(\mathrm{PO}_{4}\right)_{2} / \mathrm{l}-\mathrm{rGO}$. 

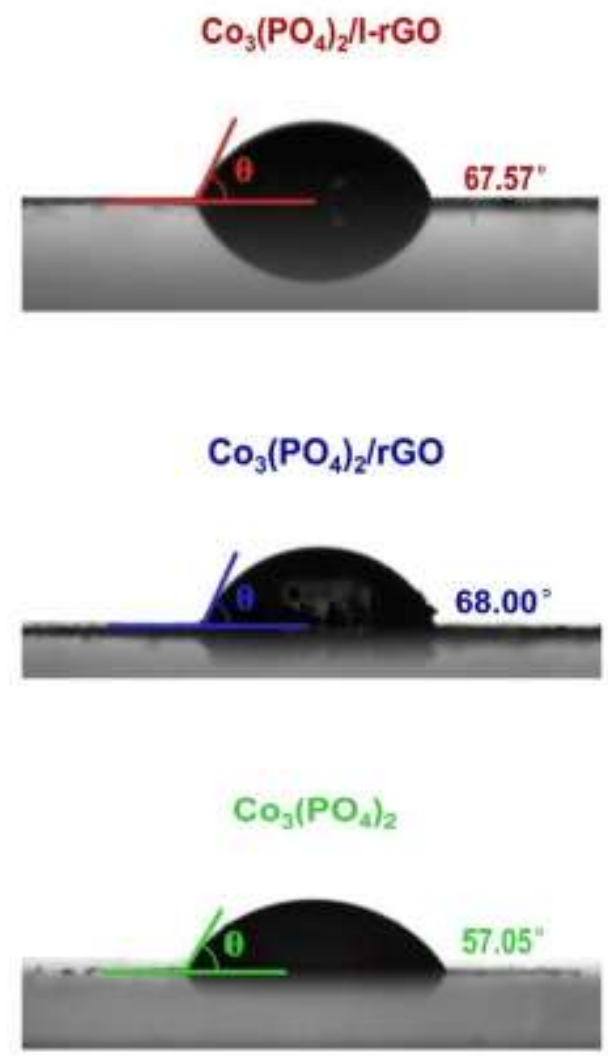

Figure S2. (a) Contact angle test of $\mathrm{Co}_{3}\left(\mathrm{PO}_{4}\right)_{2} / \mathrm{l}-\mathrm{rGO}, \mathrm{Co}_{3}\left(\mathrm{PO}_{4}\right)_{2} / \mathrm{rGO}$, and $\mathrm{Co}_{3}\left(\mathrm{PO}_{4}\right)_{2}$ after treatment with water plasma. 
Table S1. Comparison for the Electrochemical Oxidation Behaviors of the Prepared Working Electrodes toward $30 \mu \mathrm{M} \mathrm{O}_{2}{ }^{--}$.

\begin{tabular}{ccc}
\hline working electrodes & half wave potential & peak current $(\mu \mathrm{A})$ \\
& $(\mathrm{V})$ & 329.5 \\
$\mathrm{Co}_{3}\left(\mathrm{PO}_{4}\right)_{2} / \mathrm{l}-\mathrm{rGO} / \mathrm{GCE}$ & 0.68 & 215.8 \\
$\mathrm{Co}_{3}\left(\mathrm{PO}_{4}\right)_{2} / \mathrm{rGO} / \mathrm{GCE}$ & 0.73 & 65.3 \\
$\mathrm{Co}_{3}\left(\mathrm{PO}_{4}\right)_{2} / \mathrm{GCE}$ & 0.74 & \\
\hline
\end{tabular}



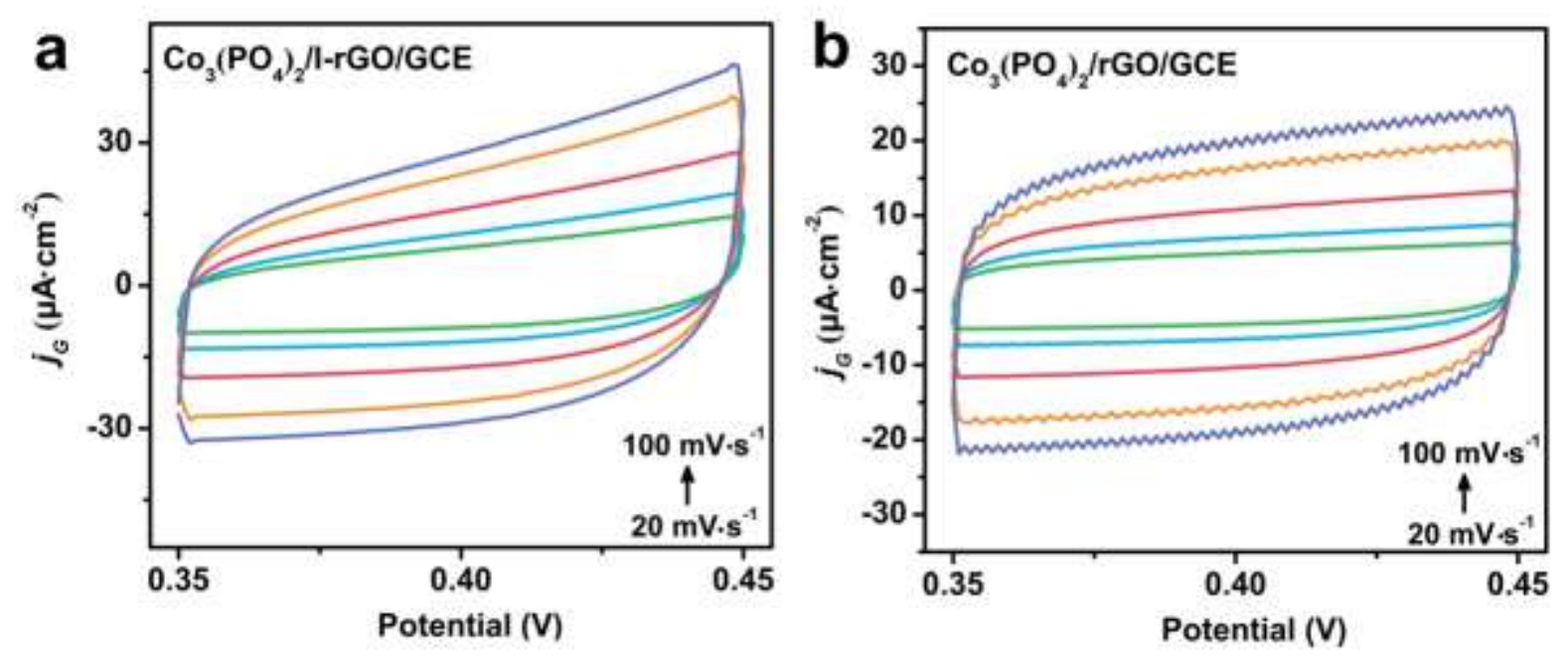

Figure S3. a, CVs of $\mathrm{Co}_{3}\left(\mathrm{PO}_{4}\right)_{2} / \mathrm{l}-\mathrm{rGO} / \mathrm{GCE}$ at various scan rate $\left(20-100 \mathrm{mV} \cdot \mathrm{s}^{-1}\right) \cdot \mathbf{b}$, $\mathrm{CVs}$ of $\mathrm{Co}_{3}\left(\mathrm{PO}_{4}\right)_{2} / \mathrm{rGO} / \mathrm{GCE}$ at various scan rate $\left(20-100 \mathrm{mV} \cdot \mathrm{s}^{-1}\right)$. 


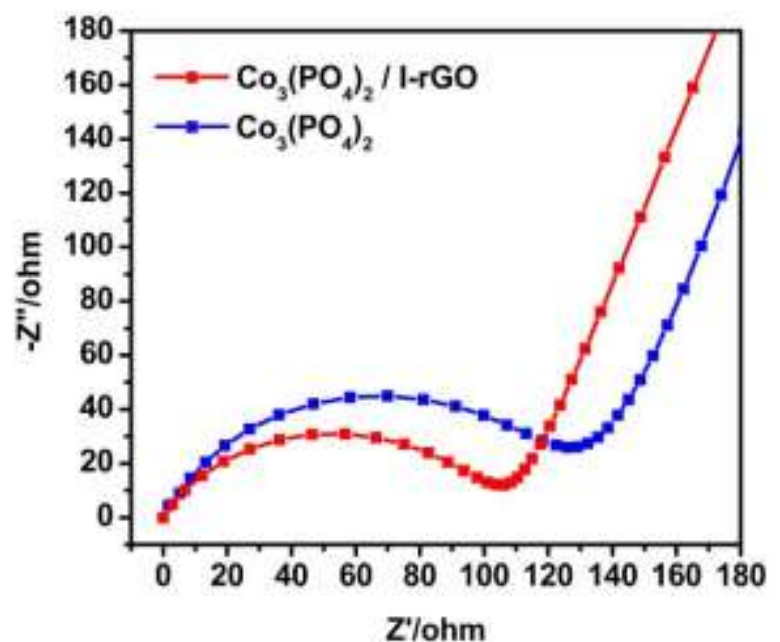

Figure S4. Nyquist plots of $\mathrm{Co}_{3}\left(\mathrm{PO}_{4}\right)_{2} / \mathrm{l}-\mathrm{rGO}$ and $\mathrm{Co}_{3}\left(\mathrm{PO}_{4}\right)_{2}$ modified electrodes. 


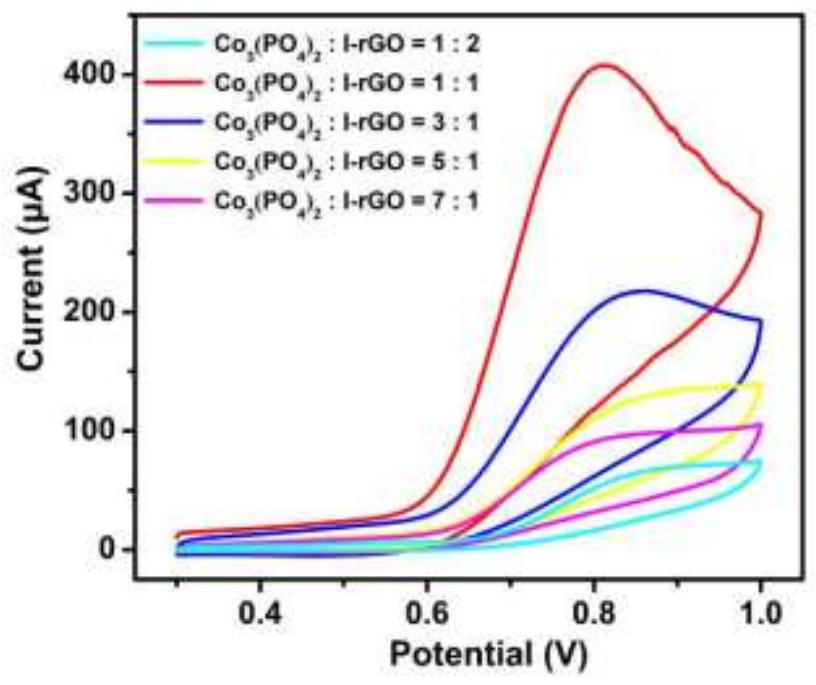

Figure S5. CVs of different ratio of $\mathrm{Co}_{3}\left(\mathrm{PO}_{4}\right)_{2}$ : I-rGO in $0.01 \mathrm{~mol} \mathrm{~L}^{-1} \mathrm{PBS}$ with $40 \mu \mathrm{M}$ $\mathrm{O}_{2}{ }^{\cdot-}$. 


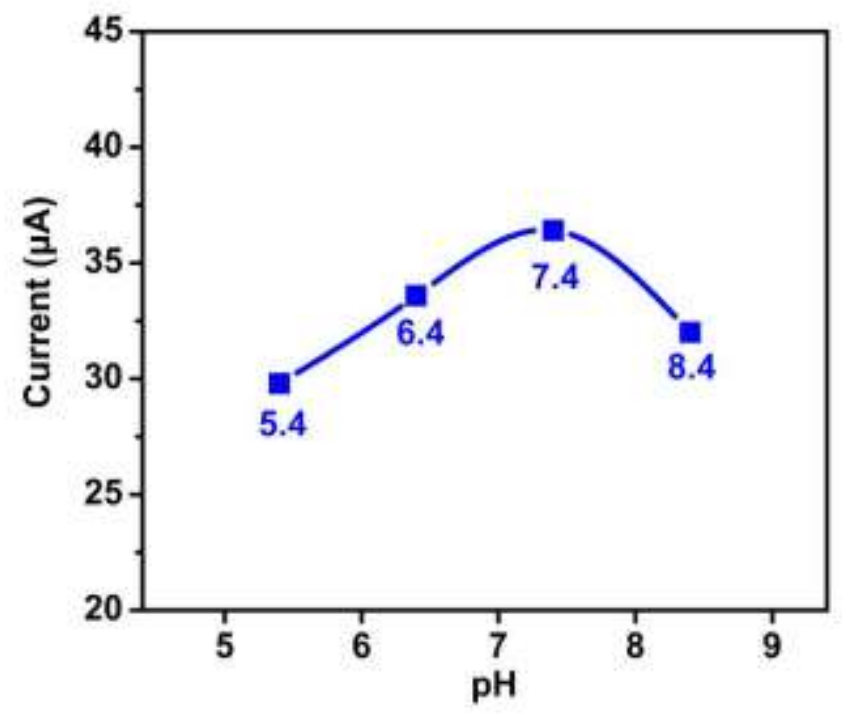

Figure S6. Plot of the dependence of the peak current on different $\mathrm{pH}$ values (from

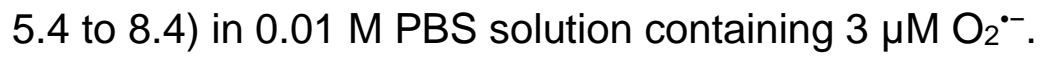




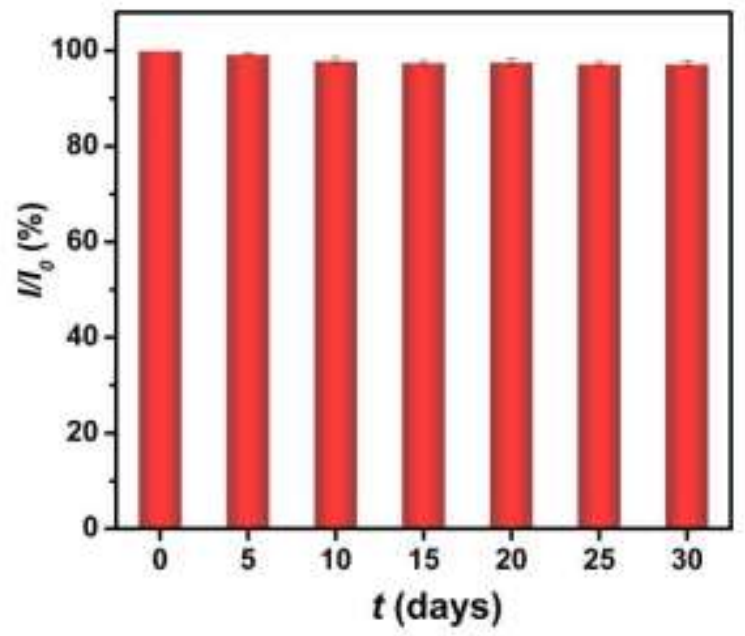

Figure S7. The stability test of the $\mathrm{CO}_{3}\left(\mathrm{PO}_{4}\right)_{2} / \mathrm{l}-\mathrm{rGO}$ sensor. 